\title{
THE SECOND NEIGHBOURHOOD FOR BIPARTITE TOURNAMENTS
}

\author{
RUIJUAN Li \\ School of Mathematical Sciences \\ Shanxi University \\ Taiyuan 030006, PR China \\ e-mail: ruijuanli@sxu.edu.cn \\ AND \\ Bin SHENG \\ College of Computer Science and Technology \\ Nanjing University of Aeronautics and Astronautics \\ Nanjing 211106, PR China \\ Department of Computer Science, Royal Holloway \\ University of London, TW20 OEX Egham, UK \\ e-mail: shengbinhello@nuaa.edu.cn
}

\begin{abstract}
Let $T(X \cup Y, A)$ be a bipartite tournament with partite sets $X, Y$ and $\operatorname{arc}$ set $A$. For any vertex $x \in X \cup Y$, the second out-neighbourhood $N^{++}(x)$ of $x$ is the set of all vertices with distance 2 from $x$. In this paper, we prove that $T$ contains at least two vertices $x$ such that $\left|N^{++}(x)\right| \geq\left|N^{+}(x)\right|$ unless $T$ is in a special class $\mathcal{B}_{1}$ of bipartite tournaments; show that $T$ contains at least a vertex $x$ such that $\left|N^{++}(x)\right| \geq\left|N^{-}(x)\right|$ and characterize the class $\mathcal{B}_{2}$ of bipartite tournaments in which there exists exactly one vertex $x$ with this property; and prove that if $|X|=|Y|$ or $|X| \geq 4|Y|$, then the bipartite tournament $T$ contains a vertex $x$ such that $\left|N^{++}(x)\right|+\left|N^{+}(x)\right| \geq 2\left|N^{-}(x)\right|$.
\end{abstract} Keywords: second out-neighbourhood, out-neighbourhood, in-neighbourhood, bipartite tournament.

2010 Mathematics Subject Classification: 05C20, 05C12, $05 \mathrm{C} 07$.

\section{REFERENCES}

[1] J. Bang-Jensen and G. Gutin, Digraphs: Theory, Algorithms and Applications, 2nd Ed. (Springer-Verlag, London, 2009). 
[2] J. Bang-Jensen and J. Huang, Quasi-transitive digraphs, J. Graph Theory 20 (1995) 141-161. doi:10.1002/jgt.3190200205

[3] G. Chen, J. Shen and R. Yuster, Second neighborhood via first neighborhood in digraphs, Ann. Combin. 7 (2003) 15-20. doi:10.1007/s000260300001

[4] Z. Cohn, A. Godbole, E. Wright Harkness and Y. Zhang, The number of Seymour vertices in random tournaments and digraphs, Graphs Combin. 32 (2016) 18051816. doi:10.1007/s00373-015-1672-9

[5] N. Dean and B.J. Latka, Squaring the tournament-an open problem, Congr. Numer. 109 (1995) 73-80.

[6] D. Fidler and R. Yuster, Remarks on the second neighborhood problem, J. Graph Theory 55 (2007) 208-220. doi:10.1002/jgt.v55:3

[7] D.C. Fisher, Squaring a tournament: a proof of Dean's conjecture, J. Graph Theory 23 (1996) 43-48. doi:10.1002/(SICI)1097-0118(199609)23:1〈43::AID-JGT4 $\rangle 3.0 . C O ; 2-K$

[8] S. Ghazal, Seymour's second neighbourhood conjecture for tournaments missing a generalized star, J. Graph Theory 71 (2012) 89-94. doi:10.1002/jgt.20634

[9] G. Gutin and R. Li, Seymour's second neighbourhood conjecture for quasi-transitive oriented graphs. arxiv.org/abs/1704.01389.

[10] F. Havet and S. Thomassé, Median orders of tournaments: a tool for the second neighborhood problem and Sumner's conjecture, J. Graph Theory 35 (2000) 244-256. doi:10.1002/1097-0118(200012)35:4〈244::AID-JGT2〉3.0.CO;2-H

[11] Y. Kaneko and S.C. Locke, The minimum degree approach for Paul Seymour's distance 2 conjecture, Congr. Numer. 148 (2001) 201-206.

[12] J.W. Moon, Solution to problem 463, Math. Mag. 35 (1962) 189. doi: $10.2307 / 2688555$

[13] B. Sullivan, A summary of results and problems related to the Caccetta-Häggkvist conjecture. arxiv.org/abs/math/0605646.

[14] R. Li and B. Sheng, The second neighbourhood for quasi-transitive oriented graphs, submitted.

Received 12 June 2017

Revised 13 October 2017 Accepted 13 October 2017 